\title{
MULTIPLE REFLECTION EFFECTS IN NONLINEAR MIXTURE MODEL FOR HYPERSPECTRAL IMAGE ANALYSIS
}

\author{
C. Y. $\operatorname{Liu}^{\mathrm{a}}, \mathrm{H} \cdot \operatorname{Ren}^{\mathrm{b}} *$ \\ ${ }^{a}$ Dept. of Computer and Information Engineering, National Central University, Taiwan - liuafrc@cc.ncu.edu.tw \\ ${ }^{\mathrm{b}}$ Center for Space and Remote Sensing Research, National Central University, Taiwan - hren@ csrsr.ncu.edu.tw
}

\section{Commission VII, WG VII/4}

\begin{abstract}
KEY WORDS: Hyperspectral images, Linear mixture model (LMM), Generalized bilinear model (GBM), Modified generalized bilinear model (MGBM)
\end{abstract}

\begin{abstract}
:
Hyperspectral spectrometers can record electromagnetic energy with hundreds or thousands of spectral channels. With such high spectral resolution, the spectral information has better capability for material identification. Because of the spatial resolution, one pixel in hyperspectral images usually covers several meters, and it may contain more than one material. Therefore, the mixture model must be considered. Linear mixture model (LMM) has been widely used for remote sensing target classifications, because of its simplicity and yields reasonable results for smooth surfaces. For rough surfaces, the physical interactions of the light scattered between multiple materials in the scene must be considered. Recently, Generalized Bilinear Model (GBM) is proposed and it includes the double reflection between different materials into a nonlinear model, but it ignores the interactions within the same material. In this study, we propose a modified version of GBM to further consider this effect in our model, called Modified Generalized Bilinear Model (MGBM).
\end{abstract}

\section{INTRODUCTION}

Hyperspectral spectrometers can record electromagnetic energy with hundreds or thousands of spectral channels. With such high spectral resolution, the spectral information has better capability for material identification. Because of the instantaneous field of view (IFOV) of one spectrum usually cover several meters, it may contain more than one material. Therefore, the mixture model must be considered. Linear mixture model (LMM) has been widely used for remote sensing target classifications (Keshava, 2002 and Manolakis, 2001), because of its simplicity and yields reasonable results for smooth surfaces. But it can be inappropriate for rough surfaces where the physical interactions of the light scattered between multiple materials in the scene. Recently, Generalized Bilinear Model (GBM) is proposed and it includes the interaction between different materials which results a nonlinear model with second order interactions between two different endmembers (Halimi, 2011a,b). In this study, we propose a modified version of GBM to further consider the multiple reflections within the same endmember, called Modified Generalized Bilinear Model (MGBM). Preliminary results show the advantage of MGBM in estimating the probability of multiple reflections. The dataset for experiment was taken from ROSIS sensor at the University of Pavia in 2001. There are 103 spectral bands after water absorption channels are removed. Three major materials in the image scene, roads, trees and grass, have different roughness which can be estimated with MGBM. Our experimental result shows the estimated abundances of these three materials are similar among LMM, GBM and MGBM. But the probability of double reflection in MGBM within trees is much higher than roads and grass, which indicates trees are much rougher than other two materials.

\section{LINEAR MIXTURE MODEL AND GENERALIZED BILINEAR MODEL}

Linear mixture model assumes the recorded spectrum is the linear combination of the spectra of all materials in the IFOV with their corresponding percentage. This assumption is suitable for smooth surface. Let $\vec{y}$ be an $L \times 1$ column vector and denote the spectral signature of one spectrum, where $L$ is the number of spectral bands. And $M$ is an $L \times p$ endmembers matrix denoted by $\left[\overrightarrow{\mathbf{m}_{1}}, \cdots, \overrightarrow{\mathbf{m}_{R}}\right]$, where $R$ is number of materials and $\overrightarrow{\mathbf{m}_{j}}$ is an $L \times 1$ column vector representing the $j$ th endmember signature resident in $\vec{y}$. Let $\boldsymbol{\alpha}=\left[\alpha_{1} \alpha_{2} \cdots \alpha_{R}\right]^{T}$ be a $p \times 1$ abundance column vector associated with $\vec{y}$ and $\overrightarrow{\mathbf{n}}$ is an additive white noise. Then LMM can be represented by following equation.

$$
\vec{y}=M \overrightarrow{\boldsymbol{\alpha}}+\overrightarrow{\mathbf{n}}
$$

For rough surface, multiple reflections must be considered. Generalized Bilinear Model considers second-order interactions between $i$-th and $j$-th endmembers (for $\mathrm{i}, j=1, \ldots, \mathrm{R}$ and $i \neq j$ ) and the observed mixed pixel $\vec{y}$ can be written as

\footnotetext{
* Corresponding author
} 


$$
\vec{y}=M \overrightarrow{\boldsymbol{\alpha}}+\sum_{i=1}^{R-1} \sum_{j=i+1}^{R} \gamma_{i, j} \alpha_{i} \alpha_{j} \vec{m}_{i} \otimes \vec{m}_{j}+\overrightarrow{\mathbf{n}}
$$

where $\otimes$ denotes that Hadamard product operation and the $\gamma_{i, j}$ is the nonlinear mixing coefficient that controls the interaction between endmembers $i$ and $j$.

\section{MODIFIED GENERALIZED BILINEAR MODEL AND FAN-FCLS ALGORITHM}

The MGBM is a nonlinear model that considers the second order interactions within the same material. The corresponding mixed pixel $\vec{y}$ is then expressed as

$$
\vec{y}=M \overrightarrow{\boldsymbol{\alpha}}+\sum_{i=1}^{R} \sum_{j=i}^{R} \gamma_{i, j} \alpha_{i} \alpha_{j} \vec{m}_{i} \otimes \vec{m}_{j}+\overrightarrow{\mathbf{n}}
$$

Because the MGBM is a nonlinear problem, it is difficult to solve. Fan-FCLS algorithm is proposed to solve the optimization problem (Fan, 2009). Estimating the MGBM parameter vector can be formulated as following optimization problem

$$
\vec{\theta}=\arg \min _{\vec{\theta}}\|\vec{y}-\vec{\mu}(\vec{\theta})\|^{2}
$$

where

$$
\vec{\mu}(\vec{\theta})=M \overrightarrow{\boldsymbol{\alpha}}+\sum_{i=1}^{R} \sum_{j=i}^{R} \gamma_{i, j} \alpha_{i} \alpha_{j} \vec{m}_{i} \otimes \vec{m}_{j}+\overrightarrow{\mathbf{n}}
$$

Since it is a nonlinear problem, Taylor series expansion is applied to solve it. At iteration $t$ of the algorithm with point estimation

$$
\vec{\theta}^{t}=\left(\alpha_{1}{ }^{t}, \ldots, \alpha_{R}{ }^{t}, \gamma_{1,1}{ }^{t}, \ldots, \gamma_{R, R}{ }^{t}\right)
$$

the linearization yields

$$
\vec{\mu}(\vec{\theta}) \approx \vec{\mu}\left(\vec{\theta}^{t}\right)+\left.\frac{\partial \vec{\mu}}{\partial \vec{\theta}}\right|_{\vec{\theta}=\vec{\theta}^{t}}\left(\vec{\theta}-\vec{\theta}^{t}\right)
$$

The iterative updating rule can be written as follow

$$
\vec{\theta}^{t+1}=\arg \min _{\vec{\theta}}\left\|\vec{h}^{t}-g^{t} \vec{\theta}\right\|^{2}
$$

and

$$
\vec{h}^{t}=\vec{y}-\vec{\mu}\left(\vec{\theta}^{t}\right)+g^{t} \vec{\theta}^{t}
$$

Secondly, the MGBM are subject to sum-to-one and nonnegative constraints. The optimization problem is applying the fully constrained least square (FCLS) algorithm. The constraints can be handled by using the extended matrix

$$
G^{t}=\left(\begin{array}{ccc}
\delta g_{L \times S}^{t} & & 0_{L \times S} \\
0_{S \times R} & I_{S \times S} & I_{S \times S} \\
1_{R \times 1}^{T} & 0_{S \times 1}^{T} & 0_{S \times 1}^{T}
\end{array}\right)
$$

and

$$
H^{t}=\left(\begin{array}{c}
\delta \vec{h}^{t} \\
1_{S} \\
1
\end{array}\right)
$$

The stopping criterion is met when the difference between the estimations of next iteration is less than a preset threshold.

\section{EXPERIMENTAL RESULT}

The data set for experiment was taken from ROSIS sensor at the University of Pavia in 2001. There are 103 spectral bands after removing water absorption channels. A subset of $35 \times 40$ pixels is selected for experiment as shown in Fig 1. There are three endmembers in this image: Road, Tree and Grass. The surfaces of these three endmembers have different roughness. Road is smooth and expect to have low multiple reflection and the tree has the roughest surface which expect to have high multiple reflection.

Figures 2, 3 and 4 are the abundance fraction maps estimated by LMM, GBM and MGBM respectively. The result shows the abundance estimation of Road, Tree and Grass are similar among these three models. Figure 3 also shows the secondorder reflection between different materials for GBM, and this phenomenon highly appears at the boundary of two materials. MGBM further considers the second-order reflection within the same material as shown in Figure 4. The color bars beside the fraction maps indicate the higher possibility of multiple reflections for Tree than Road and Grass, which confirms our expectation.

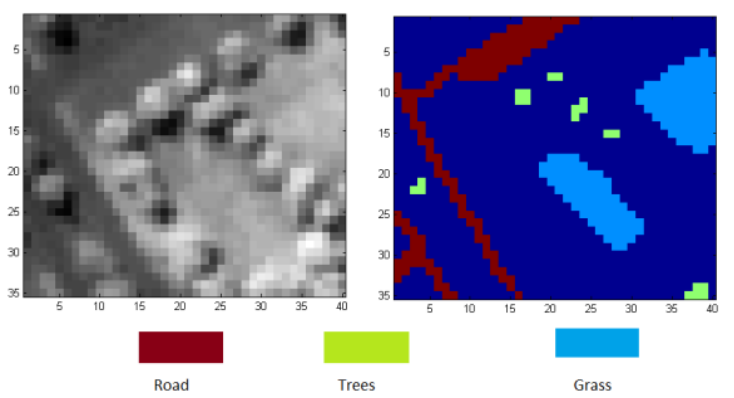

Figure 1. Hyperspectral image with ground truth in University of Pavia 

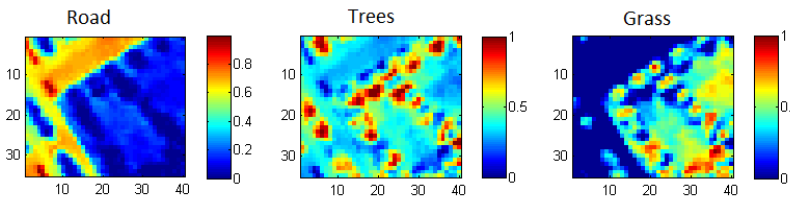

Figure 2. Fraction maps estimated using LMM
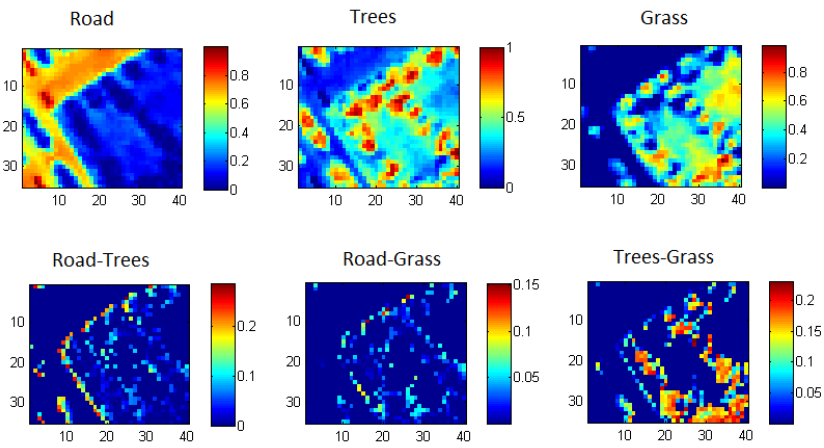

Figure 3. Fraction maps estimated using GBM
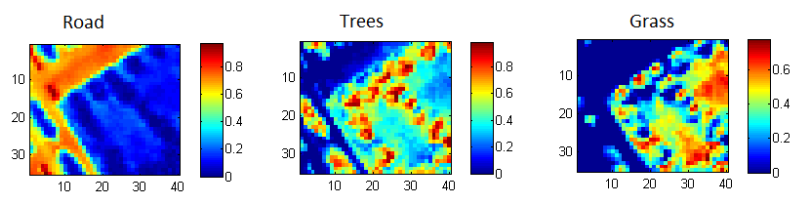

Road-Trees


Road-Road

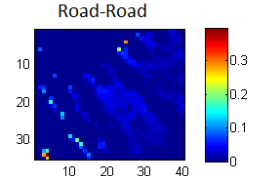

Trees-Trees
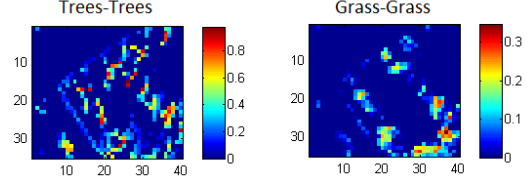

Figure 4. Fraction maps estimated using MGBM

\section{CONCLUSION}

Mixture model has been widely implemented for remote sensing for several decades. The Linear Mixture Model neglects multiple reflections and assumes linear combination which is suitable for smooth surface. However, the interaction between materials has to be considered for rough surface. The proposed Modified Generalized Bilinear Model is developed from Generalized Bilinear Model by further include second order reflection within the same material, and the preliminary results support our assumption. The experiment with in situ measurement under controlled environment will be conducted and analyzed in the near future.

\section{ACKNOWLEDGEMENT}

The authors would like to express their appreciation to the Ministry of Science and Technology in Taiwan for the research fund under the contract 104-2119-M-008-022.

\section{REFERENCES}

Fan, W., Hu, B., Miller, J., Li, M., 2009, Comparative Study Between a New Nonlinear Model and Common Linear Model for Analysing Laboratory Simulated-forest Hyperspectral Data. International Journal of Remote Sensing. 30(11): p. 2951-2962.

Halimi A., Altmann Y., Dobigeon N., Tourneret J.-Y., 2011a, Nonlinear unmixing of hyperspectral images using a generalized bilinear model, Statistical Signal Processing Workshop (SSP), (49) 4153-4162.

Halimi A., Altmann Y., Dobigeon N., Tourneret J.-Y., 2011b, Unmixing hyperspectral images using the generalized bilinear model, Geoscience and Remote Sensing Symposium (IGARSS), 2011 IEEE, 1886 - 1889.

Keshava N., Mustard J.F., 2002, Spectral unmixing, IEEE Signal Processing Magazine, 44-57.

Manolakis D., Siracusa C., Shaw G., 2001, Hyperspectral subpixel target detection using the linear mixing model, IEEE Trans. Geosci. Remote Sens, (39) 1392-1409. 